\title{
CAN WE STOMACH COX-2 INHIBITORS?
}

\author{
A Higham, Consultant Physician \\ Royal Lancaster Infirmary
}

\section{INTRODUCTION}

The last twenty years have seen major changes in the field of gastroenterology. The development of $\mathrm{H}_{2}$-receptor antagonists revolutionised the treatment of peptic ulcer disease but these have been superseded by the proton pump inhibitors. The rediscovery of Helicobacter pylori (H pylori) and the demonstration that this organism plays a causal role in peptic ulcer disease has led to further significant changes in the practice of gastroenterology worldwide. Despite this, the mortality from upper gastrointestinal haemorrhage has remained practically unchanged. Most recently a new class of non-steroidal anti-inflammatory drug (NSAID), the selective inhibitors of cyclo-oxygenase-2 (COX-2), has appeared and has been heralded as a major advance on the standard NSAIDs. Whilst their main use is in the treatment of arthritic disease it may be that they represent a further major advance for the gastroenterologist. This article will review the effects of NSAIDs on the stomach, the physiology of COX-1 and $\mathrm{COX}-2$, and their role in peptic ulcer disease and the potential benefits of selective COX-2 inhibition.

\section{STANDARD NSAIDS AND PEPTIC ULCER DISEASE}

Ever since the demonstration that there is a dose/response relationship between the ingestion of aspirin and the incidence of peptic ulcers, NSAIDs have been associated with gastric and duodenal ulcer disease. Almost $30 \%$ of patients taking NSAIDs regularly will have endoscopic evidence of gastric and/or duodenal ulceration (Figure 1) although the annual incidence of clinically important GI

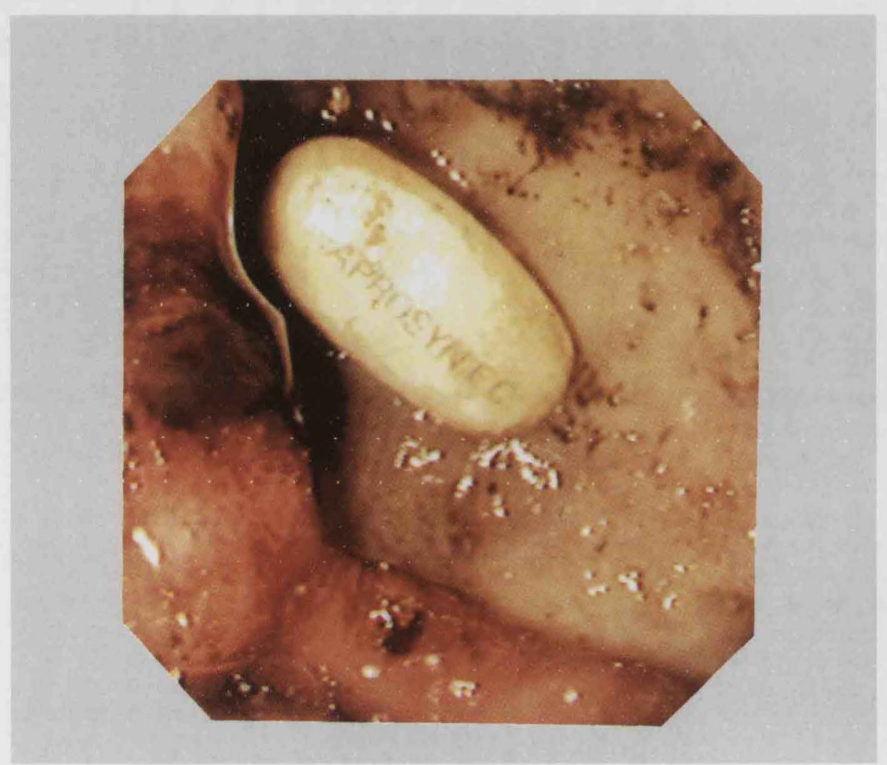

Figure I Endoscopic view of prepyloric ulcer showing Naprosyn EC in ulcer base events (ie those requiring hospitalisation or referral for endoscopy) is approximately $2 \%$.

Nevertheless there are approximately 4000 deaths per annum from peptic ulcer disease in patients over 60 years of age. Approximately one quarter of ulcer complications (haemorrhage or perforation) occur in patients taking NSAIDs and it is therefore likely that 1000 deaths per annum in patients over 60 are due to ingestion of standard NSAIDs. The selective COX-2 inhibitors were developed with the rationale that they should be as clinically effective as NSAIDs without the propensity for ulcerogenesis in the stomach and duodenum. To understand this rationale more fully, there follows a brief review of the physiology of gastric mucosal protection and the COX-1 and COX-2 enzymes.

\section{MECHANISM OF GASTRIC MUCOSAL PROTECTION}

The mucosal layer of the stomach is constantly being exposed to noxious agents resulting in minor but ongoing damage. As a consequence of this the gastric mucosa deploys a number of both constitutive and stimulated regeneration pathways which together maintain the integrity of the mucosal barrier. The major components of this maintenance programme are the continual growth of the epithelial cell layer, constant production of the mucous bicarbonate barrier and maintenance of its hydrophobicity.

All of these components are critically dependant on regulated gastric blood supply and on the continued production of prostaglandins within the gastric mucosa. The local production of prostaglandins within the gastric mucosa is controlled by the action of cyclo-oxygenase.

\section{PHYSIOLOGY OF COX-1 AND COX-2}

Cyclo-oxygenase exists in at least two isoforms. Both enzymes convert arachidonic acid to prostaglandin $\mathrm{H} 2$. Arachidonic acid itself is produced by the action of phospholipases on membrane phospholipid that is released whenever cell injury or tissue damage occurs. Prostoglandin $\mathrm{H} 2$ is then converted into a variety of different prostaglandins depending on the tissue specific production of intermediate enzymes involved in this process.

COX-1 is constitutively expressed and is, therefore, being constantly produced at low levels in many tissues throughout the body including the gastric mucosa and platelets. This ensures constant and steady production of prostaglandins essential for gastric mucosal protection, epithelial cell surface restitution and platelet aggregation. $\mathrm{COX}-1$ therefore plays a key role in the continual maintenance of gastric mucosal integrity. 
Conversely, COX-2 expression is inducible and in normal circumstances the protein is not produced. Under certain conditions, most notably inflammation, gene expression is induced and rapidly upregulated so that COX-2 protein is produced in large quantities and available arachidonic acid is preferentially converted into prostaglandins such as PGE2, which modulate pain and further promote the inflammatory response.

Standard NSAIDs such as indomethacin, ibuprofen, naproxen and diclofenac inhibit both $\mathrm{COX}-1$ and COX-2 indiscriminately thereby causing deleterious side effects such as gastric mucosal damage as well as anti-inflammatory effects. Selective COX-2 inhibition should theoretically have the same anti-inflammatory and analgesic effects whilst avoiding undesirable gastrointestinal toxicity as this is the result of COX-1 inhibition. Numerous studies, including large randomised controlled trials, have now been performed in order to prove the therapeutic advantage of COX-2 inhibition over standard NSAID treatment. There is general agreement that the COX-2 inhibitors are as good as standard NSAIDs in relieving the symptoms of arthritis and the therapeutic advantage of these drugs relies almost solely on their limited GI side-effect profile.

\section{COX-2 INHIBITION AND THE STOMACH}

Several studies have shown that in the healthy human stomach there is no detectable expression of the COX-2 gene or protein ${ }^{(1,2)}$. In keeping with this is the demonstration that even large doses (up to 10 times normal) have no discernible effect on the integrity of gastric mucosa assessed endoscopically after one week's treatment ${ }^{(3)}$. This is in sharp contrast to the effects on the gastric mucosa of one week's treatment with high doses of standard NSAIDs (Figure 2).

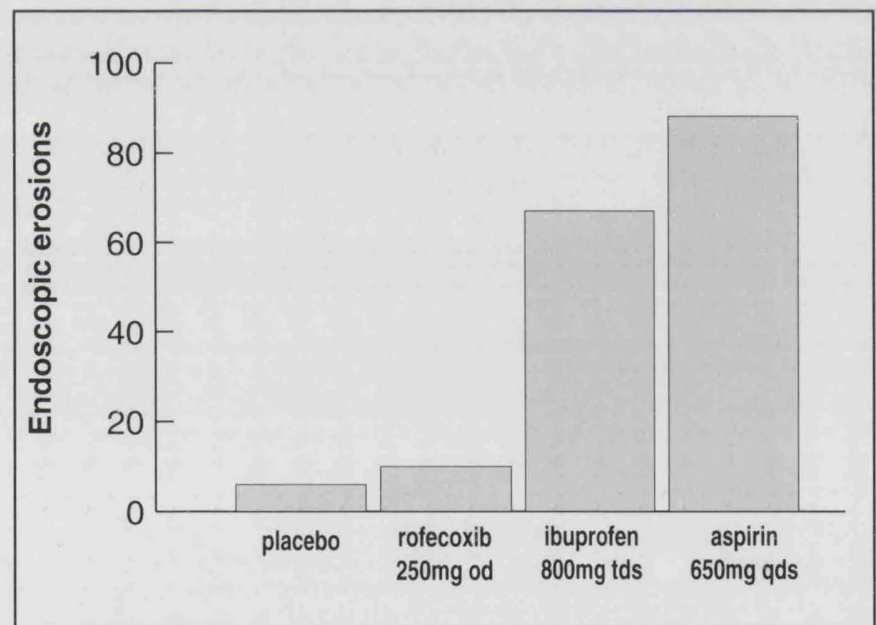

Figure 2 Average number of erosions detected endoscopically after one week's treatment with rofecoxib, ibuprofen, aspirin or placebo at stated doses. Although the doses of standard NSAIDs are large, the dose of rofecoxib is approximately ten times the standard therapeutic dose.

Despite this the incidence of erosions is equivalent to placebo.

Confirmatory evidence for the absence of a role for COX-2 in the healthy stomach is provided by the ex vivo analysis of gastric mucosal prostaglandin synthesis after oral dosing of either a standard NSAID or the highly specific COX-2 inhibitor rofecoxib. Prostaglandin production was unaffected by rofecoxib but greatly reduced by conventional NSAID treatment ${ }^{(4)}$. Whilst this provides powerful evidence in favour of COX-2 inhibitors and their inability to cause ulcers, there is no doubt that ulcers and complicated upper GI events still occur in patients taking these drugs in the setting of a large randomised controlled trial ${ }^{(5)}$. Having said that, the rates of ulcer, perforation and bleeding in these trials are significantly reduced (Figure 3 ) and statistically equivalent to placebo $^{(6)}$

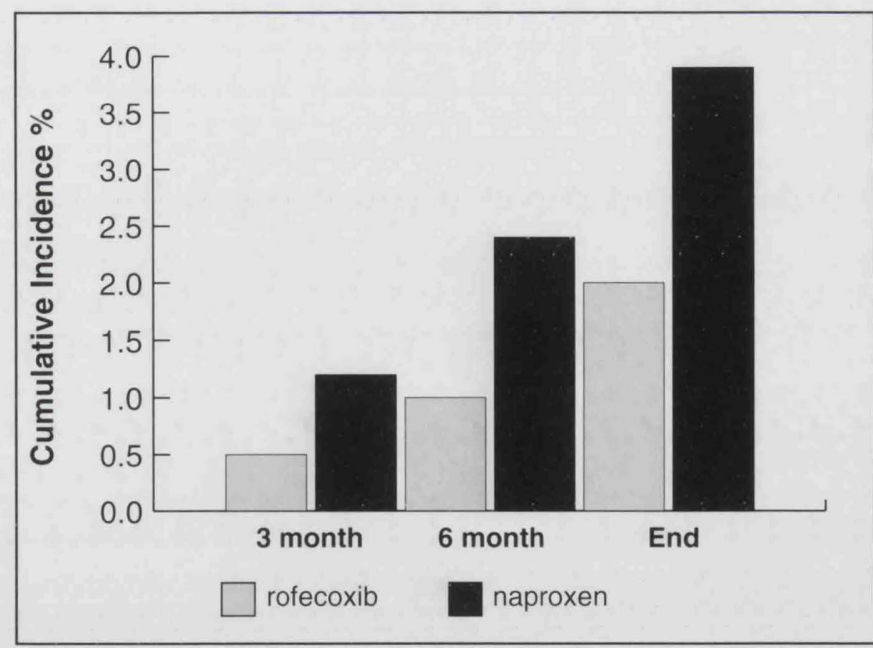

Figure 3 The data show the cumulative incidence of upper GI events in patients taking either rofecoxib or naproxen for up to 12 months for symptomatic relief of osteoarthritis. The data are adapted from the VIGOR study. There are significantly fewer upper GI events in patients takeing rofecoxib.

In the large randomised trials there was no randomisation or subgroup analysis according to the $H$ pylori status of the people taking part. Given that this is the leading risk factor for ulcer development it is the most likely explanation for the ulcer rate in both placebo and COX-2 arms of the trial. Concomitant smoking and alcohol consumption may play a lesser part in ulcer development. In fact there are very few studies that have examined the role of $H$ pylori status in the development of ulcers in patients taking COX-2 inhibitors Such an argument is supported by the results of Emery et al who analysed the rates of ulcer development in $H$ pyloripositive and -negative patients taking either NSAID or the selective COX-2 inhibitor celecoxib (Figure 4).

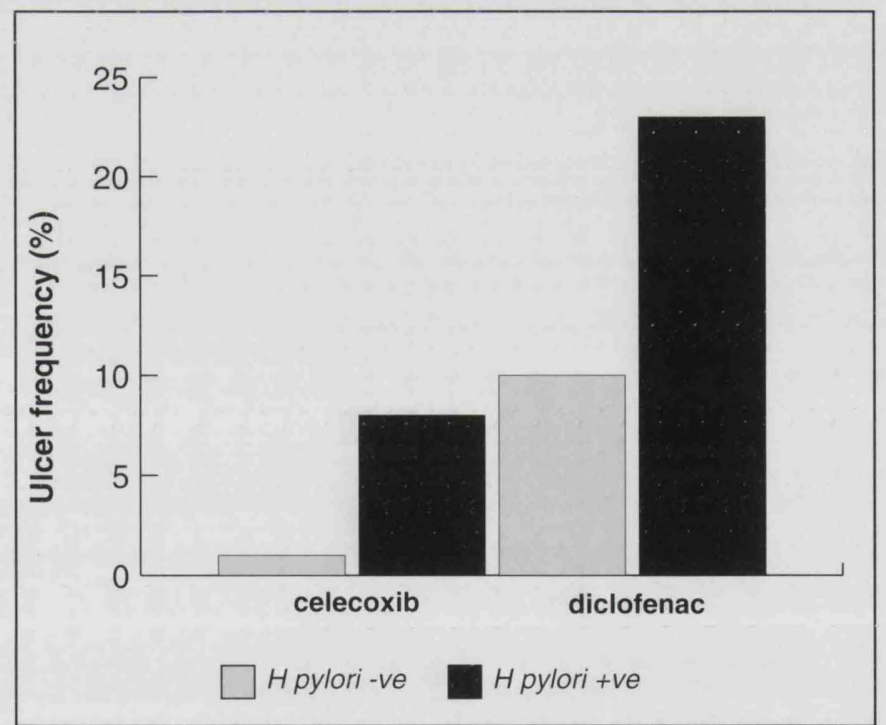

Figure 4 Ulcer frequency according to $\mathrm{H}$ pylori status in patients taking either celecoxib or diclofenac. The reduction in ulcer frequency in $\mathrm{H}$ pylorinegative patients compared to $\mathrm{H}$ pylori-positive patients taking diclofenac

has not been borne out by other larger trials. Note the remarkably low incidence of ulcers in $\mathrm{H}$ pylori-negative patients taking the selective $\mathrm{COX}-2$ inhibitor celecoxib. Data adapted from Emery et al

Almost all the ulcers in patients taking celecoxib occurred in those that were $H$ pylori-positive. Could such ulcers be prevented by prior $H$ pylori eradication therapy? The question remains to be answered and there is little data available to resolve the issue at present. Immunohistochemical studies suggest that $\mathrm{COX}-2$ protein at 
least is still detectable up to one year after eradication therapy. Whether the protein is functional and still being produced this long after successful eradication therapy remains to be clarified.

\section{COX-2 INHIBITION AND THE HEART}

Despite their proven effectiveness in reducing upper GI events there are concerns regarding potential cardiotoxicity which have been highlighted by the National Institute for Clinical Excellence $(\mathrm{NICE})^{(8)}$ and which may limit their potential therapeutic use. The VIGOR study ${ }^{(5)}$ showed a statistically significant excess of cardiovascular events in patients taking rofecoxib compared to naproxen. Low-dose aspirin for cardiovascular prophylaxis was not permitted in either group. Perhaps the simplest explanation for this result is again a demonstration of the highly selective action of rofecoxib on COX-2, whilst amongst the NDSAIDs Naprosyn has a spectrum of activity closest to that of aspirin. The Naprosyn group were therefore effectively receiving cardiovascular prohylaxis whereas those taking rofecoxib were not. Previous studies with rofecoxib have not shown this effect, though it is hard to dismiss the results of VIGOR as a statistical quirk. More importantly, the production of protective (anti-thrombotic) prostacyclins within vascular endothelium is dependent in part on $\mathrm{COX}-2$ activity so that inhibition of this action may directly increase the risk of cardiovascular events.

It is hoped that further research will determine the overall cardiovascular risk of taking a COX-2 inhibitor. In the interim, it is clear that low-dose aspirin does increase the risk of ulcer disease and there is no evidence as yet to support the rationale for taking a COX-2 inhibitor along side low-dose aspirin in an attempt to reduce ulcer rates in patients requiring NSAID treatment. It is, however, also true that taking a standard NSAID in addition to low-dose aspirin further increases the risk of ulcer disease.

\section{CONCLUSION}

The COX-2 inhibitors have fulfilled their expected potential given the rationale for their development. They have no effect on the healthy stomach and do not cause peptic ulceration. Ulcers still occur in patients taking COX-2 inhibitors but at rates similar to the general population. The role and timing of eradication therapy in $H$ pylori-positive patients who require an NSAID and may benefit from a COX-2 inhibitor remain to be determined. Newer anti-platelet agents that provide cardiovascular prophylaxis without effecting cyclooxygenase activity may overcome the current difficulties in prescribing either a standard NSAID or a COX-2 inhibitor in those patients requiring cardiovascular prophylaxis.

\section{REFERENCES}

1 Localisation of cyclo-oxygenase 1 and 2 in Helicobacter pylori-related gastritis and gastric ulcer tissues in humans. Tatsuguchi A, Sakamoto C, Wada K et al. Gut 2000;46:782789.

2 Helicobacter pylori infection induces cyclo-oxygenase-2 expression in human gastric mucosa. Sawaoka H, Kawano S, Tsuji S et al. Prostaglandins Leukot Essent Fatty Acids 1998;59:313-316.

3 Specific inhibition of cyclo-oxygenase-2 with MK-0966 is associated with less gastroduodenal damage than either aspirin or ibuprofen. Lanza FL, Rack MF, Simon TJ et al. Aliment Pharmacol Ther 1999;13:761-767.

4 Rofecoxib, a COX-2 inhibitor, does not inhibit human gastric muscosal prostaglandin production. Wright NJ, Gottesdiener K, Garlick NM et al. Gastroenterol 2001;120(4):867-873.

5 Comparison of upper gastrointestinal toxicity of rofecoxib and naproxen in patients with rheumatoid arthritis. Bombadier C, Laine L, Reicin A et al. New Engl J Med 2000;343:1520-1528.

6 Treatment of osteoarthritis with celecoxib, a cyclooxygenase- 2 inhibitor: a randomised controlled trial. Bensen WG, Fiechtner JJ, McMillen JI et al. Mayo Clin Proc 1999;74:1095-1105.

7 Celecoxib versus diclofenac in long-term management of rheumatoid arthritis: randomised double-blind comparison. Emery P, Zeidler H, Kvien TK et al. Lancet 1999;354:21062111.

8 National Institute for Clinical Excellence: Technology appraisal guidance No 27, July 2001. www.nice.org.uk 